\title{
Correction to: Control for multifunctionality: bioinspired control based on feeding in Aplysia californica
}

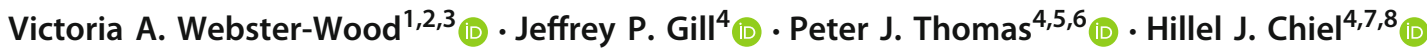

Published online: 23 February 2021

(c) Springer-Verlag GmbH Germany, part of Springer Nature 2021

\section{Correction to: \\ Biological Cybernetics (2020) 114:557-588 https://doi.org/10.1007/s00422-020-00851-9}

The authors have identified a typesetting error in their representation of the frictional forces in the original version of this manuscript. This correction has been provided to address this error to allow reproduction of the linked code. The code for the model and the results are not impacted by this error. Calculation of the frictional forces is done correctly in the code available on GitHub. The authors sincerely apologize for any confusion this error may have caused.

The original article can be found online at https://doi.org/10.1007/ s00422-020-00851-9.

Victoria A. Webster-Wood

vwebster@andrew.cmu.edu

1 Department of Mechanical Engineering, Carnegie Mellon University, 5000 Forbes Ave., Pittsburgh, PA 15213, USA

2 Department of Biomedical Engineering, Carnegie Mellon University, 5000 Forbes Ave., Pittsburgh, PA 15213, USA

3 McGowan Institute for Regenerative Medicine, Carnegie Mellon University, 5000 Forbes Ave., Pittsburgh, PA 15213, USA

4 Department of Biology, Case Western Reserve University, 2080 Adelbert Road, Cleveland, OH 44106-7080, USA

5 Department of Mathematics, Applied Mathematics and Statistics, Case Western Reserve University, 10900 Euclid Avenue, Cleveland, OH 44106-4901, USA

6 Department of Electrical Computer and Systems Engineering, Case Western Reserve University, 10900 Euclid Avenue, Cleveland, OH 44106-4901, USA

7 Department of Neurosciences, Case Western Reserve University, 2080 Adelbert Road, Cleveland, OH 44106-7080, USA

8 Department of Biomedical Engineering, Case Western Reserve University, 2080 Adelbert Road, Cleveland, $\mathrm{OH}$ 44106-7080, USA
In Appendix A.5, Eqs. (91) and (92) should read:

$\left|F_{f, g}\right|=\left|F_{\mathrm{I} 2}+F_{s p, g}-F_{\mathrm{I} 3}-F_{\text {hinge }}\right|$

$\left|F_{f, g}\right|=\left|\mu_{k, g} F_{\mathrm{I} 4}\right|$

Similarly, Eqs. (101) and (102) should read:

$\left|F_{f, h}\right|=\left|F_{s p, h}+F_{f, g}\right|$

$\left|F_{f, h}\right|=\left|\mu_{k, h} F_{13, \text { ant. }}\right|$

This ensures that the signs of the frictional forces during swallowing and rejection are calculated correctly. It should be noted that the components of $F_{\mathrm{I} 3 \text {,ant. in Eq. (102) are }}$ always positive, but the magnitude indication is included here for clarity of sign definition. As indicated in the original manuscript, these frictional forces only affect the head and grasper during swallowing, as the tube during rejection is not fixed to the force transducer and provides no reaction force. Additionally, they are not applied during biting as no seaweed is present. See the provided code for details of implementation within the semi-implicit framework for each of the three distinct behaviors.

Publisher's Note Springer Nature remains neutral with regard to jurisdictional claims in published maps and institutional affiliations. 